\title{
Nurses' Perceived Barriers to and Facilitators of Pain Assessment and Management in Critical Care Patients: A Systematic Review
}

\section{Mohammad Rababa iD Shatha Al-Sabbah Audai A Hayajneh (1)}

Adult Health Nursing Department, Jordan University of Science and Technology, Irbid, Jordan
Correspondence: Mohammad Rababa Adult Health Nursing Department, Jordan University of Science and Technology, Irbid, Jordan

Emailmjrababa@just.edu.jo

\begin{abstract}
Aim: This review aims to examine nurses' perceived barriers to and facilitators of pain assessment and management in adult critical care patients.

Background: Pain is one of the worst memories among critically ill patients. However, pain among those patients is still undertreated due to several barriers that impede effective management. Therefore, addressing the perceived barriers and facilitators to pain assessment management among critical care nurses is crucial.
\end{abstract}

Methods: A systematic search of pain assessment and management in critical care patientrelevant literature from four databases was done, following the Preferred Reporting Items for Systematic Reviews and Meta-Analyses (PRISMA) guidelines.

Results: The barriers and facilitators were categorized into four groups: nurse-related, patient-related, physician-related, and system-related. The most frequently reported barriers in this study included nurses' lack of knowledge regarding the use of pain assessment tools, patients' inability to communicate, physicians' prescription of analgesics being independent of pain scores evaluation, and absence of standardized guidelines and protocols for pain evaluation and control. For the facilitators, the most reported ones include ongoing education and professional training related to pain assessment and management, patients' ability to self-report pain, effective collaboration between physicians and nurses, and productive discussion of patients' pain scores during nurse-to-nurse handovers.

Conclusion: Various barriers and facilitators to pain assessment and management were identified and examined in this review. However, future research is still needed to further investigate these barriers and facilitators and examine any other potential associated factors among critical care nurses.

Relevance to Clinical Practice: The findings of our study could help hospital managers in developing continuous education and staff development training programs on assessing and managing pain for critical care patients. Also, our findings could be used to develop an evidence-based standard pain management protocol tailored to effectively assess and promptly treat pain in critical care patients.

Keywords: critical care nurses, pain assessment, pain management, barriers, facilitators, enablers, challenges

\section{Introduction}

For intensive care unit (ICU) patients, pain experienced during ICU stay is one of the most upsetting memories. ${ }^{1}$ According to Puntillo et al, more than half of ICU patients in 28 European countries were found to have experienced pain, whilst in the United States (US), about $60 \%$ of ICU patients reported that their pain persisted 
after discharge from the hospital. ${ }^{1}$ In addition to various physiological sources of pain, routine nursing care procedures, such as position-changing and endotracheal suctioning, are major sources of intrinsic pain for ICU patients. ${ }^{2}$ Untreated pain among critical care patients has many adverse effects, leading to serious physiological and psychological complications and increased length of critical care unit stay. ${ }^{2}$ For example, the influence of unrelieved pain on the physiological condition of patients includes hemodynamic instability, such as blood pressure elevation, tachycardia, tachypnea, and hyperglycemia. Other associated negative physical consequences include the increase in catecholamine release, immunosuppression, urinary retention, and the increase in metabolic rate. $^{2}$ Furthermore, psychological and emotional distress may develop among patients who suffer from pain as a result of their inability to cope effectively with pain. In turn, this may lower patients' quality of life. ${ }^{2}$ As a result of pain symptoms, patients may often find themselves unable to accept certain job tasks and assignments, which may lead to them losing their jobs. This may have several negative consequences on the psychological status of patients. ${ }^{3}$ Further, unrelieved pain may also negatively affect patients' social interactions, as the decline in physical and mental functioning caused by persistent pain contributes greatly to impaired social relationships and interactions. ${ }^{3}$ Thus, pain decreases individuals' quality of life and markedly decreases their mental and physical health status. ${ }^{3}$ Therefore, ICU patients require adequate pain assessment and management. However, there are several barriers to effective pain assessment and management among ICU patients. ${ }^{4}$ For example, although pain is a highly subjective experience, ICU patients' deteriorated level of consciousness and intubation limits their ability to communicate, which complicates their pain assessment and management. ${ }^{4}$

In addition to patient-related barriers, other barriers related to healthcare providers and systems complicate pain assessment and management among adult critical care patients. ${ }^{5}$ For example, studies have found nurses and physicians to have low levels of knowledge, poor attitudes, and inadequate training related to pain assessment and management for adult critical care patients. ${ }^{6}$ Furthermore, heavy workloads, high patient-provider ratios, tight work schedules, and the unavailability of standard pain protocols in adult critical care units are factors that contribute to the issue of unrelieved pain. ${ }^{5}$
Moreover, consensus is yet to be reached on the most effective pain assessment tools and pain management measures for adult critical care patients. The American Society for Pain Management Nursing recommends relying on pain-related behaviors to evaluate and treat pain among intubated patients or patients with communication deficits. ${ }^{4}$ Several nonverbal scales have been approved as valid tools for pain assessment among adult critically ill patients, such as the Behavioral Pain Scale (BPS) and Critical-Care Pain Observation Tool (CPOT). ${ }^{7}$ However, such tools have been limited by their low specificity and sensitivity for pain indicators, particularly in nonverbal patients, as well as nurses' misunderstanding, misinterpretation, and underestimation of pain behaviors and nurses' poor knowledge and attitudes related to the use of such tools. ${ }^{7}$ Physiological measures, such as blood pressure, heart rate, and respiratory rate, are sometimes used as alternative pain indicators and can provide important clues for proper pain assessment. ${ }^{8}$ However, the 2012 Society of Critical Care Medicine (SCCM) guidelines do not recommend the use of these measures alone for pain evaluation in critically ill adult patients; rather, these measures should be used in combination with the evaluation of behavioral indicators of pain. ${ }^{2}$

Pain management for critically ill adult patients in ICUs is classified into pharmacologic and nonpharmacologic treatments. ${ }^{1}$ Analgesics are the first-line therapy for pain. ${ }^{9}$ However, the use of analgesics has many adverse effects, including dizziness, physical dependence, vomiting, intolerance, respiratory depression, delayed extubation, induced bowel dysfunction, increased length of hospitalization and healthcare costs, and increased morbidity and mortality. ${ }^{9}$ According to Gaskin and Richard, ${ }^{10}$ the extra annual costs of pain management on the healthcare system in the US were between \$261$\$ 300$ billion in 2010. Several nonpharmacological therapies for pain are cost-effective, easy-to-use, and free of adverse effects. The guidelines for the Prevention and Management of Pain, Agitation/Sedation, Delirium, Immobility, and Sleep Disruption (PADIS) recommend the use of nonpharmacological interventions, such as music therapy/calming voice and massaging, to target pain among critically ill adults and minimize the harmful side effects of the recurrent and long-term use of analgesics. ${ }^{11}$

Pain assessment and management in adult ICU patients has been gaining research attention for over 25 years. ${ }^{1}$ However, pain is still a serious health problem 
around the world and is still untreated or undertreated among adult ICU patients. ${ }^{1,2}$ Unfortunately, pain experienced by adult ICU patients remains widely underrecognized and undertreated due to various barriers. ${ }^{2}$ Furthermore, there remains a wide gap between the findings of previous research and clinical practice. Moreover, there is no evidence in the nursing literature regarding the barriers and facilitators of pain assessment and management in adult critical care adult patients as perceived by nurses. Therefore, summarizing and synthesizing the existing research on pain assessment and management among adult critical care adult patients is needed to guide clinical practice and future research.

\section{Aim}

Accordingly, this review aims to identify nurses' perceived barriers to and facilitators of pain assessment and management in adult critical care patients.

\section{Materials and Methods Study Design}

The current study employed a systematic review design utilizing structured questions retrieved after reviewing the nursing literature relevant to pain among adult critical care patients. The researchers of the current review carefully evaluated and assessed the selected studies and compared their findings to reach consensus and avoid incongruities. This systematic review was guided by the following question: what are the patient-, nurse-, and system-related barriers and facilitators which impact pain assessment and management in adult critical care patients?

\section{Inclusion Criteria}

The studies included in the review were (1) peer-reviewed quantitative or qualitative studies, (2) published in English in the last ten years, (3) conducted among critical care nurses, and (4) related to the barriers and facilitators of adequate pain assessment and management in adult critical care patients.

\section{Exclusion Criteria}

Studies which were (1) dissertations, (2) book chapters, (3) conducted on target populations other than nurses, (4) conducted in clinical settings other than adult critical care units, or (5) written in languages other than English were excluded from this systematic review.

\section{Search Strategy}

An electronic search was performed by two of the researchers to identify all qualitative and quantitative published articles which discussed nurses' perceived barriers to and facilitators of pain assessment and management in adult critical care patients. The review was conducted in April 2021. No search specifications related to publication date were set, and the researchers thoroughly searched the following databases: EBSCO, MEDLINE, CINAHL, and PubMed. The following keywords were used: "nurse", "critical care nurses", "pain", "critically ill patients", "adult", "mechanically ventilated patients", "critical care unit", "intensive care unit", "knowledge", "attitude", "pain assessment", "pain management", "barriers", "facilitators", "enablers", and "challenges".

The researchers used the Boolean operators "AND" and "OR" to expand the search options and search the literature in more depth. The search of articles was limited to qualitative and quantitative research studies published in English and with the abstract and reference list available, using "title/" or "abstract" in the search field. After an initial search using different combinations of the previously mentioned keywords, 1053 studies were identified, and after removing exact duplicates or overlapping studies, 290 articles remained. After applying the inclusion and exclusion criteria, the retrieved articles were reduced to 50. The reference lists of all 50 articles were reviewed to identify any other relevant studies not identified through the database searches. Then, after a careful review of the abstracts of the articles in order to determine the main objectives and keywords of the studies and whether pain assessment and management in critical care patients was the main theme, a total of twenty studies were selected for review. Figure 1 below shows the Preferred Reporting Items for Meta-Analysis (PRISMA) checklist and flowchart used as a method of screening and selecting the studies eligible for the current review.

\section{Quality of Evidence Appraisal Assessment}

The researchers assessed the selected studies using the guidelines of Melnyk and Fineout-Overholt. ${ }^{12}$ Two of the researchers carried out a detailed review of the guidelines and independently evaluated each study until an agreement on each study's quality [level of evidence (LOE)]was reached.

\section{Data Synthesis}

The researchers developed an evidence-based table to summarize the detailed information of the selected studies 


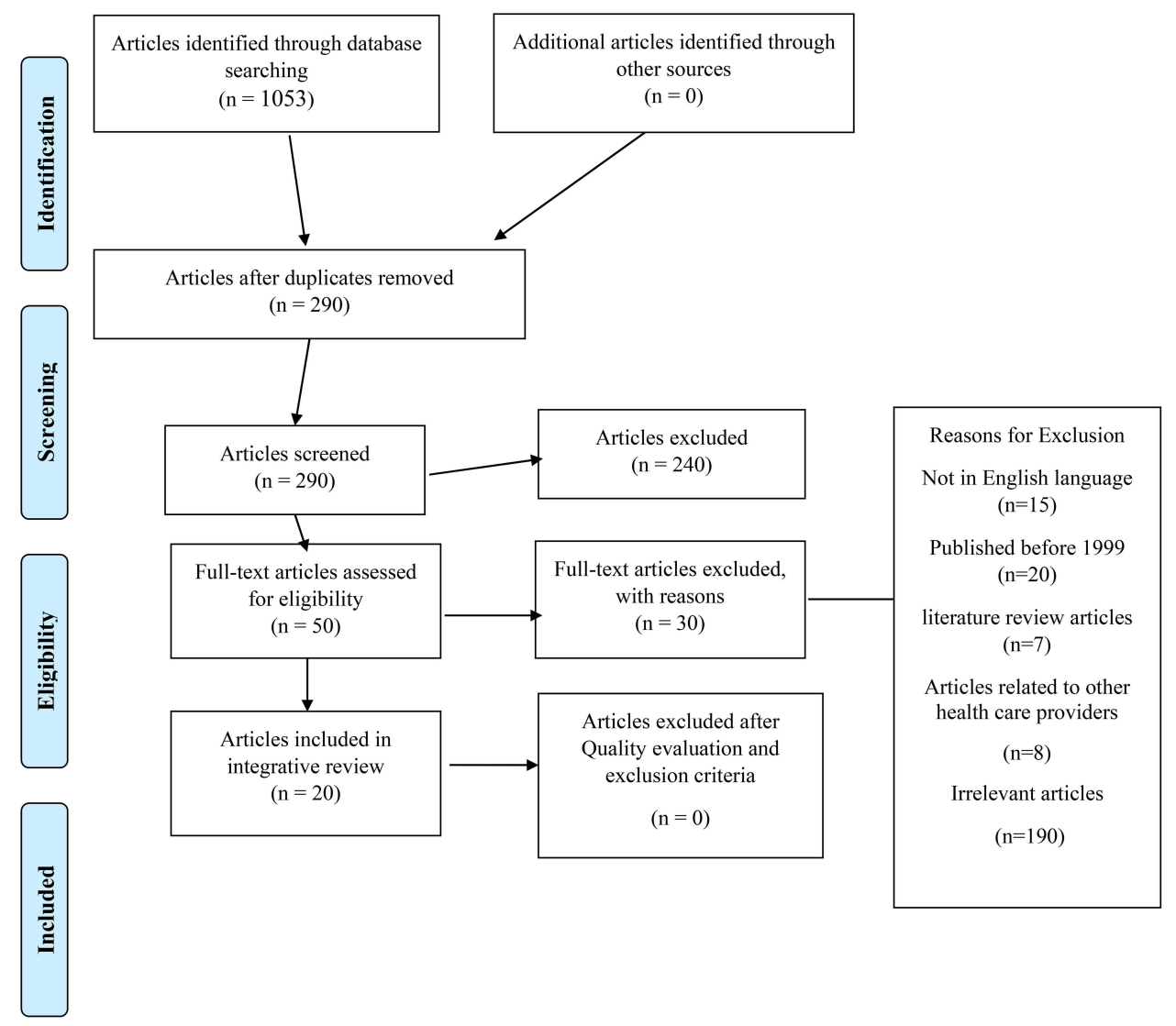

Figure I PRISMA flow chart.

Note: Adapted from: Liberati A, Altman D, Tetzlaff J, et al. The PRISMA statement for reporting systematic reviews and meta-analyses of studies that evaluate health care interventions: explanation and elaboration. Journal of Clinical Epidemiology. 2009;62(10)el-e34. Creative Commons.. ${ }^{62}$

(Table 1). The following steps were taken to carry out data synthesis: (1) description of the selected studies in terms of the aims of the study, design, population, level of evidence, and major results; (2) identification of the limitations and strengths of the selected articles; and (3) investigation of the main study results to identify heterogeneity and homogeneity.

\section{Results}

\section{Description of the Selected Studies}

The selected 20 articles were found to employ different methodologies. Four studies were qualitative, ${ }^{13-16}$ six were correlational, $^{17-22}$ three were quasi-experimental, ${ }^{23-25}$ six were descriptive, ${ }^{26-31}$ and one was longitudinal. ${ }^{21}$ The included studies were conducted on critical care patients admitted to various clinical settings, including cardiac units, ${ }^{13,18}$ oncology units, ${ }^{28}$ emergency critical care units, ${ }^{29}$ and neurosurgical units. ${ }^{22}$ In addition to the abovementioned clinical settings, the majority of the studies also included other settings, such as medical-surgical units, medical units, and burn units. ${ }^{21,27}$ Only Wang and Tsai ${ }^{20}$ and Asman et al ${ }^{17}$ were conducted in medical centers.

The proportion of female participants in the selected studies ranged from $40 \%$ to $99.7 \%$, while the proportion of male participants ranged from $14.3 \%$ to $63.2 \%$. Six of the studies were conducted in European countries. $^{13,15,16,21,26,32}$ Of the remaining studies, five were conducted in Asia, ${ }^{14,17,20,27,28}$ five in North America, ${ }^{18,19,22,23,25}$ and four in East Africa. ${ }^{24,29-31}$ Critical care nurses' pain assessment and management practices used among intensive care unit patients were measured using the Pain Assessment and Management for the Critically Ill survey in three of the studies. ${ }^{18,19,27}$ In all three of these studies, a panel of experts had reviewed the instrument's clarity, content validity, and reliability. To measure critical care nurses' knowledge of and attitudes towards pain evaluation and management, four studies used the Nurses' Knowledge and Attitudes 


\begin{tabular}{|c|c|c|c|c|c|c|c|}
\hline ه్ & $\geq$ & $\geq$ & $>$ & $\geq$ & $>$ & $\geq$ & $>$ \\
\hline 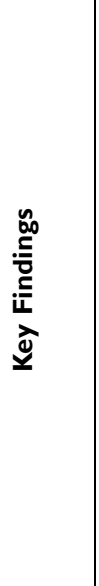 & 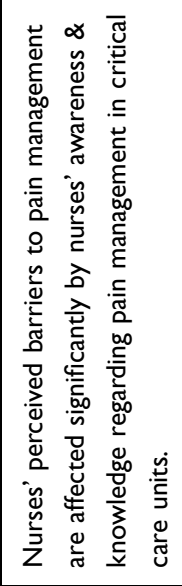 & 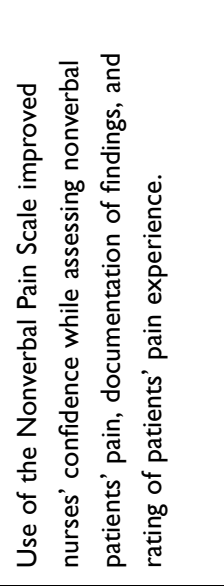 & 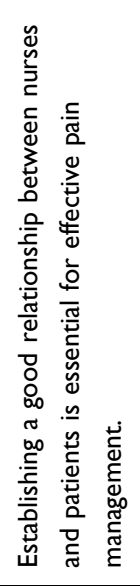 & 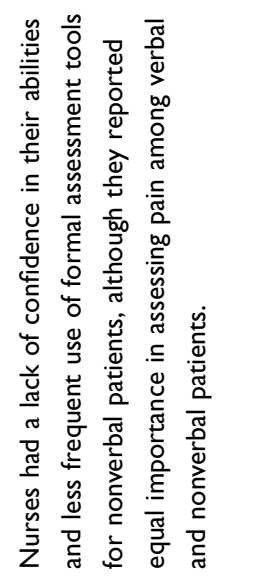 & 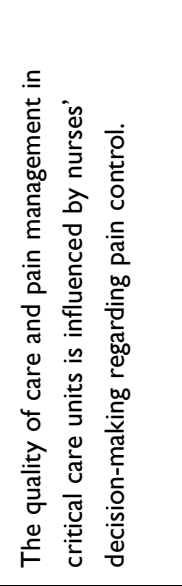 & 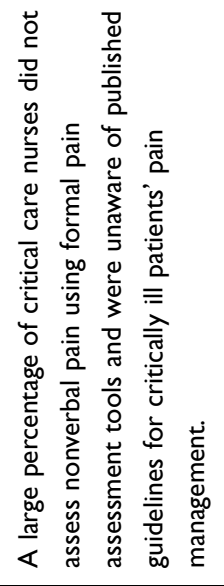 & 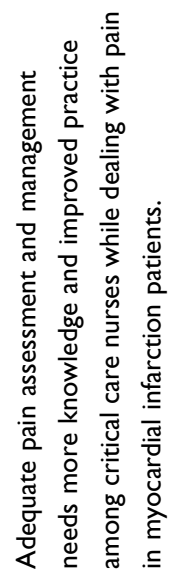 \\
\hline 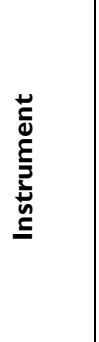 & 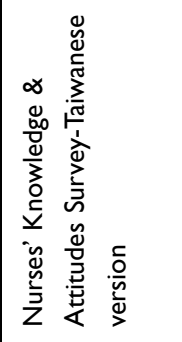 & 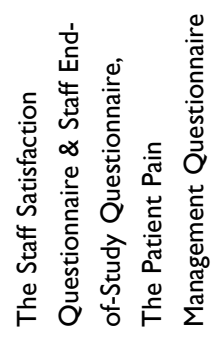 & 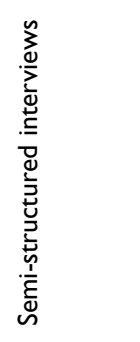 & 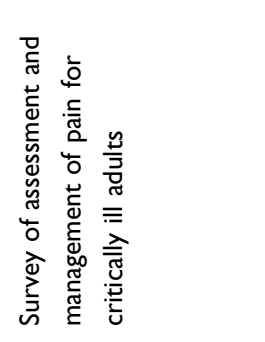 & 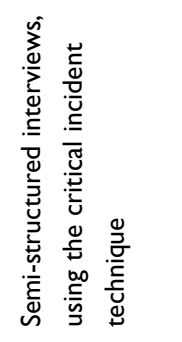 & 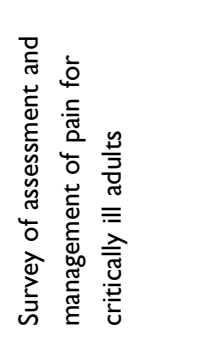 & 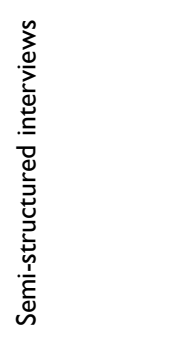 \\
\hline 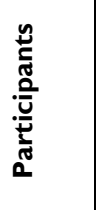 & 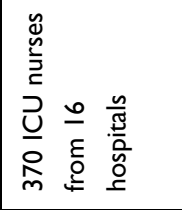 & 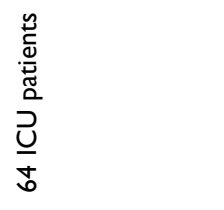 & 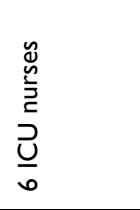 & 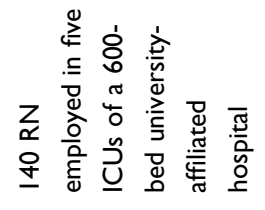 & 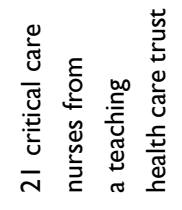 & 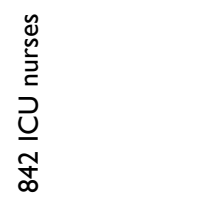 & 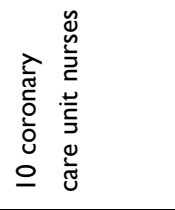 \\
\hline 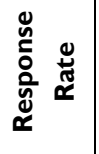 & 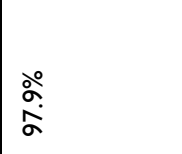 & ڤ̊ & $\underset{\substack{\stackrel{\circ}{\infty} \\
\infty}}{ }$ & ฉ̊ & ○े & $\stackrel{\stackrel{\leftrightarrow}{\sim}}{\circ}$ & ㅇㅇㅇ \\
\hline 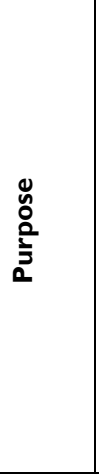 & 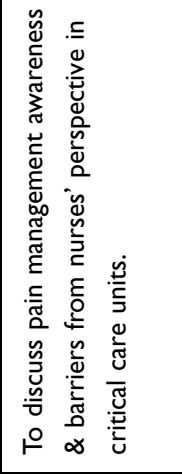 & 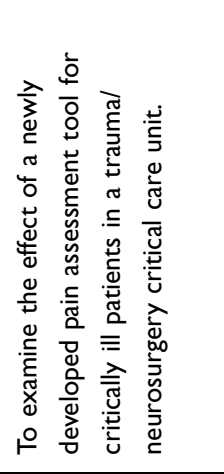 & 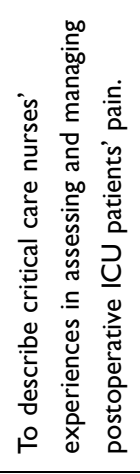 & 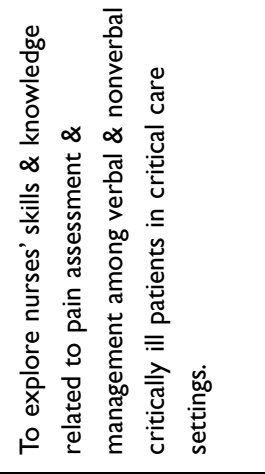 & 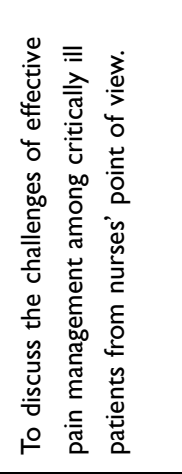 & 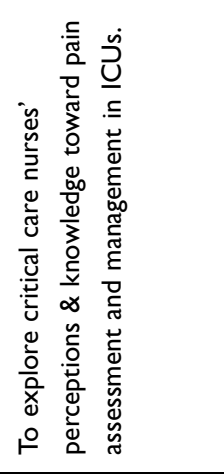 & 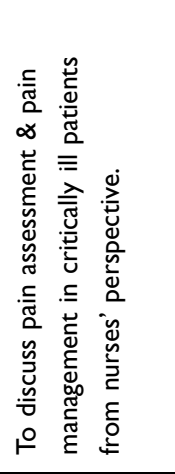 \\
\hline 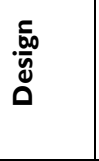 & 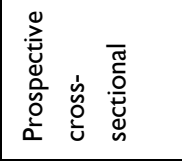 & 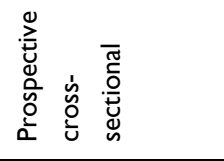 & 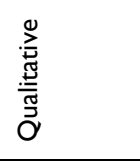 & 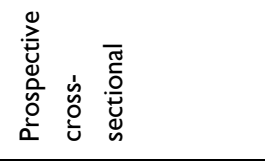 & 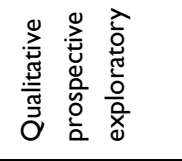 & 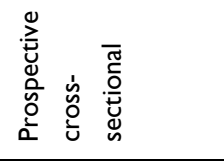 & 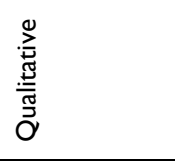 \\
\hline 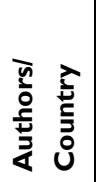 & 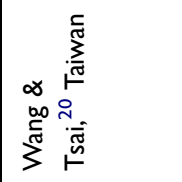 & 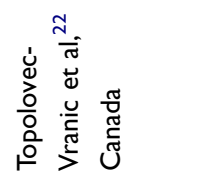 & 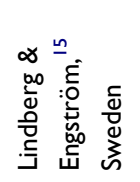 & 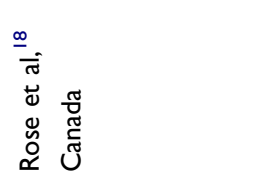 & 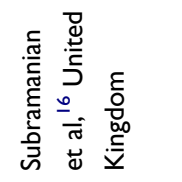 & 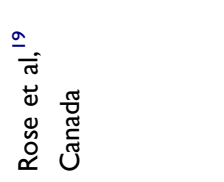 & 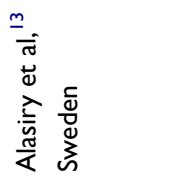 \\
\hline
\end{tabular}




\begin{tabular}{|c|c|c|c|c|c|c|}
\hline 山 & $\equiv$ & $\equiv$ & $>$ & $>$ & $\equiv$ & $>$ \\
\hline 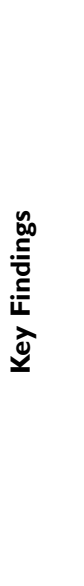 & 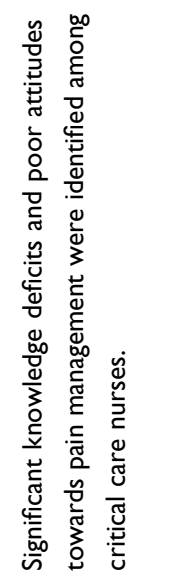 & 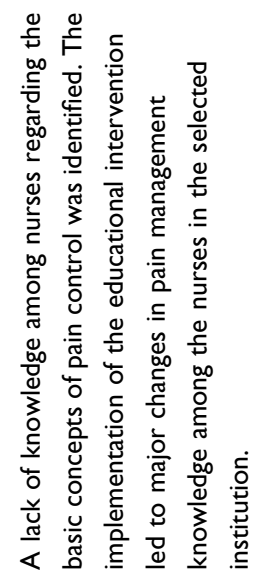 & 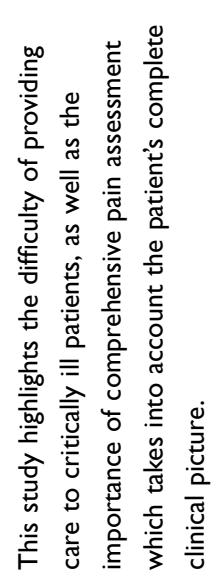 & 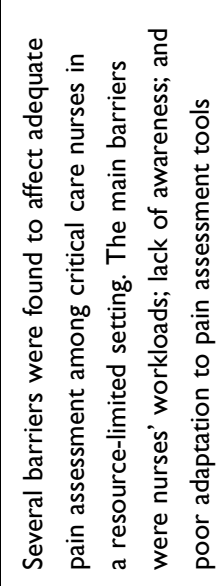 & 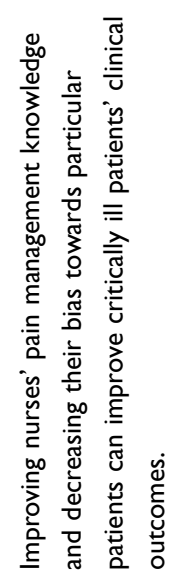 & 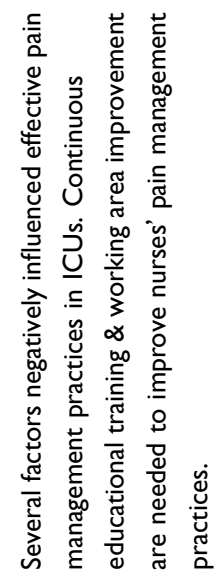 \\
\hline 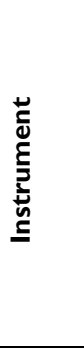 & 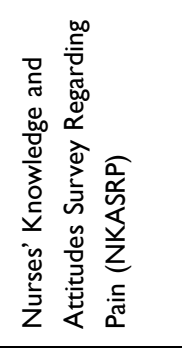 & 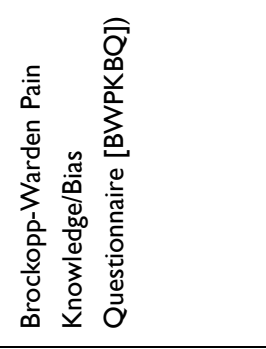 & 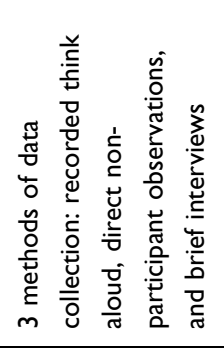 & 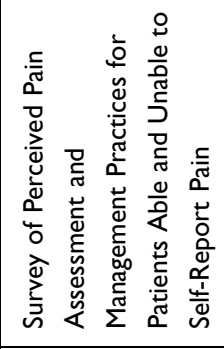 & 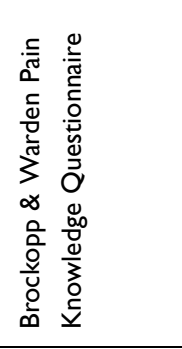 & 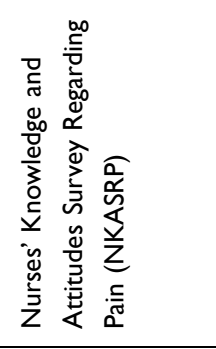 \\
\hline 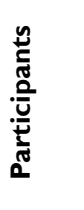 & 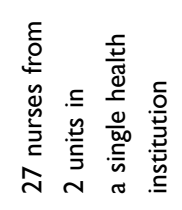 & 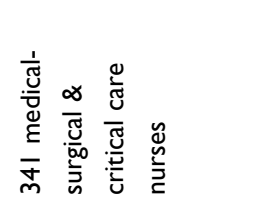 & 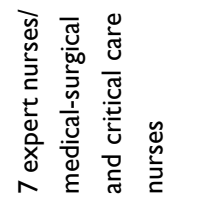 & 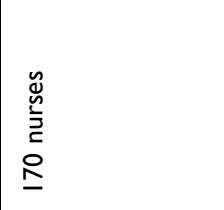 & 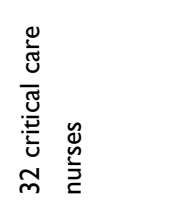 & 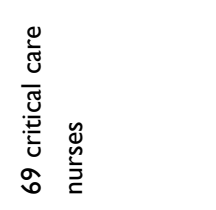 \\
\hline 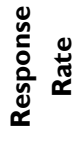 & $\frac{\stackrel{\circ}{\grave{~}}}{\stackrel{\infty}{c}}$ & ठ으 & ઠ̊ํํ & 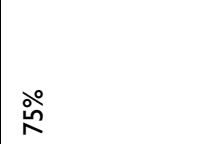 & ই̀ & ठ̊ํ \\
\hline & 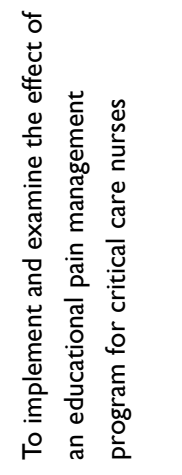 & 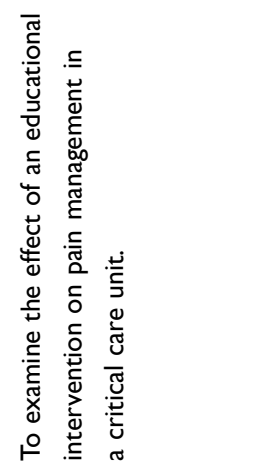 & 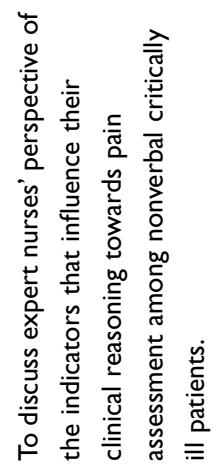 & 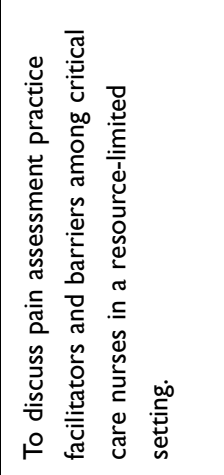 & 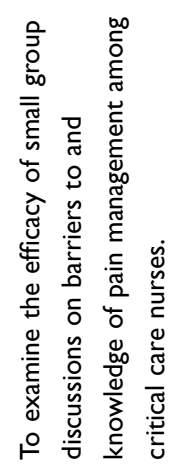 & 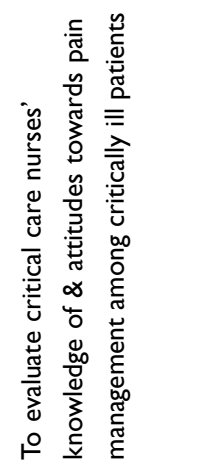 \\
\hline$\frac{.50}{\bar{\Delta}}$ & 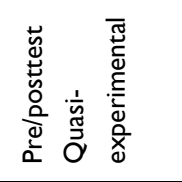 & 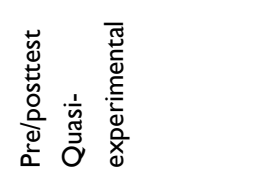 & 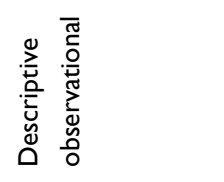 & 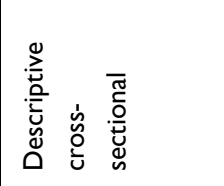 & 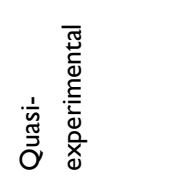 & 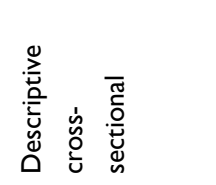 \\
\hline 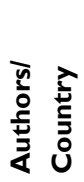 & 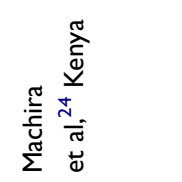 & 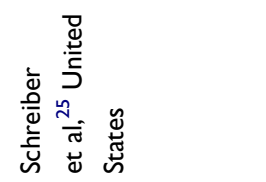 & 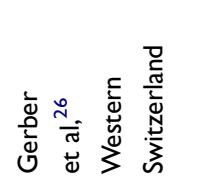 & 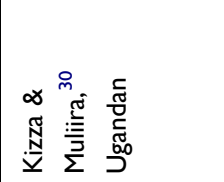 & 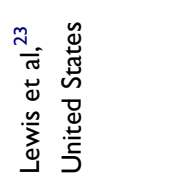 & 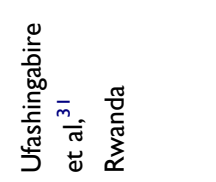 \\
\hline
\end{tabular}




\begin{tabular}{|c|c|c|c|c|c|c|}
\hline$>$ & $>$ & $>$ & $\geq$ & $\geq$ & $>$ & \\
\hline 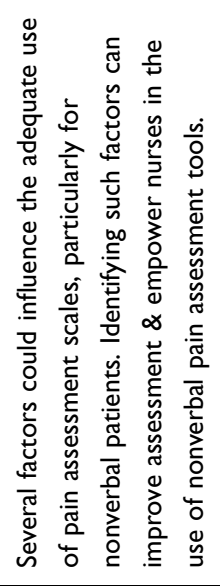 & 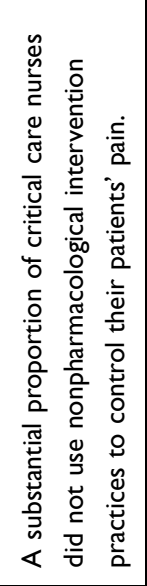 & 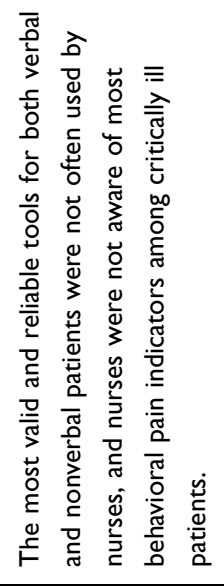 & 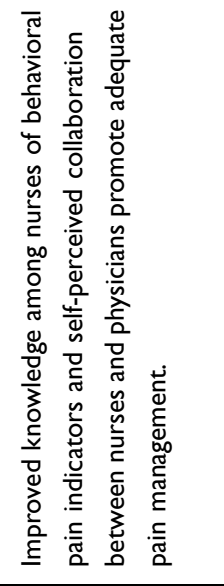 & 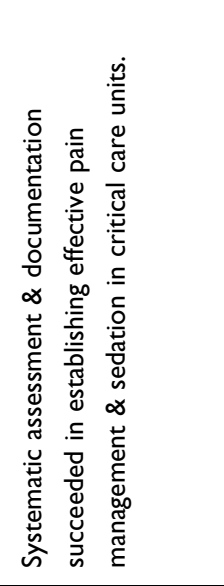 & 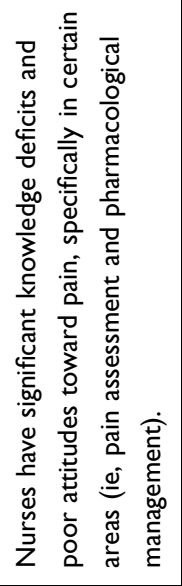 & 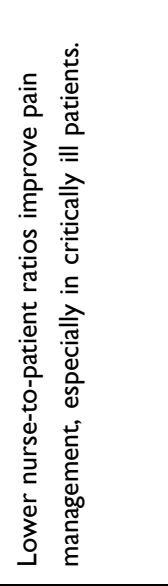 \\
\hline 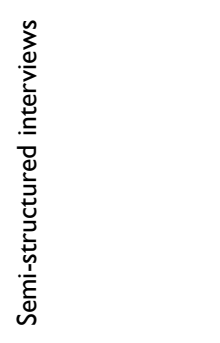 & 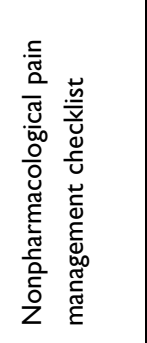 & 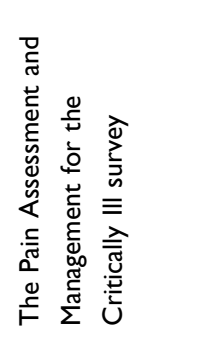 & 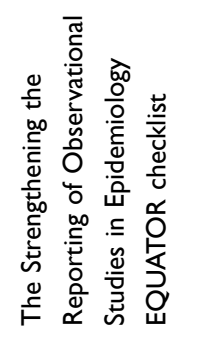 & 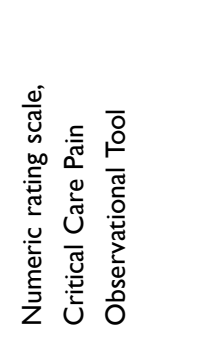 & 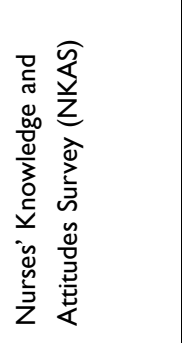 & 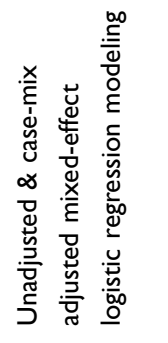 \\
\hline 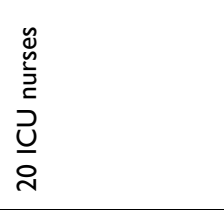 & 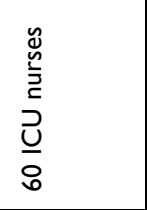 & 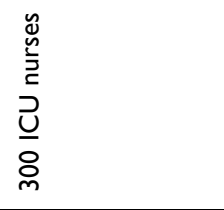 & 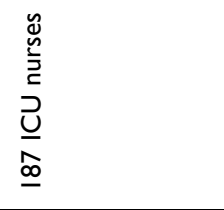 & 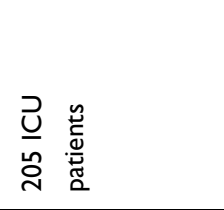 & 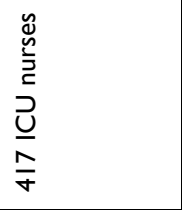 & 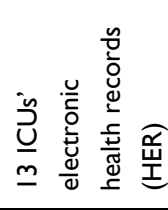 \\
\hline ळ̊으 & 응 & $\frac{\circ 0}{\circ}$ & $\begin{array}{l}\stackrel{\circ}{0} \\
\stackrel{0}{\infty} \\
\dot{\infty}\end{array}$ & $\frac{\partial 0}{\infty}$ & 。ㅇ & $\begin{array}{l}\stackrel{\circ}{\hat{n}} \\
\underline{\operatorname{n}}\end{array}$ \\
\hline 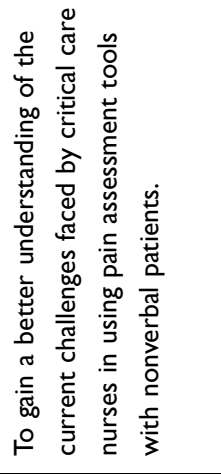 & 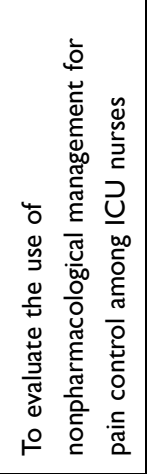 & 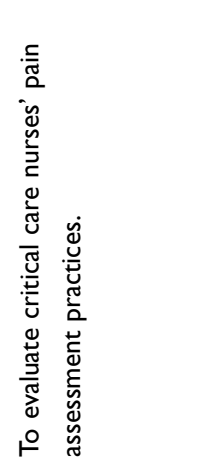 & 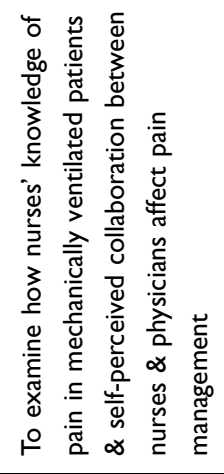 & 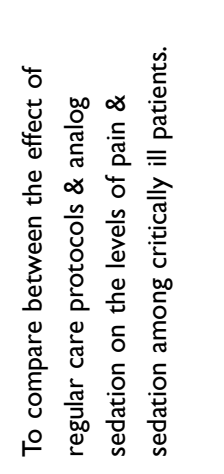 & 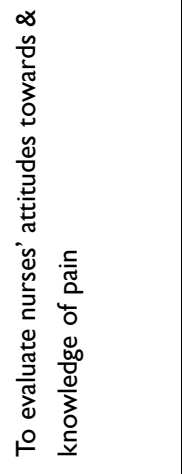 & 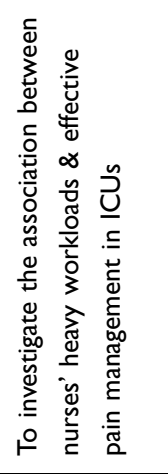 \\
\hline 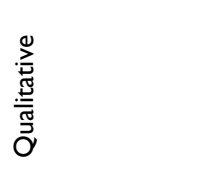 & 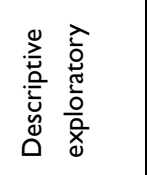 & 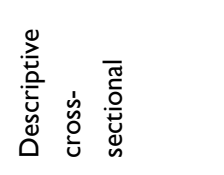 & 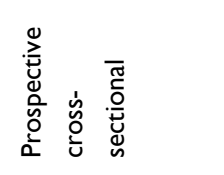 & 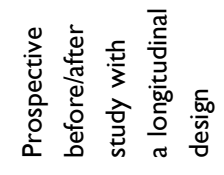 & 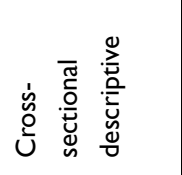 & 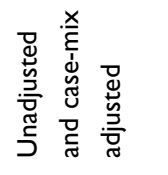 \\
\hline 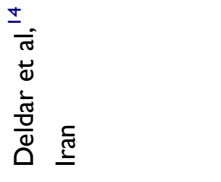 & 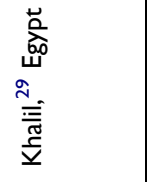 & 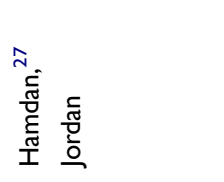 & 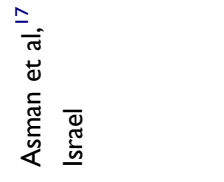 & 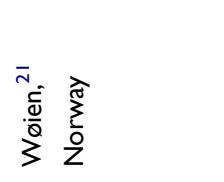 & 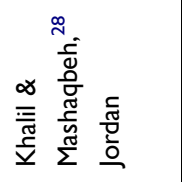 & 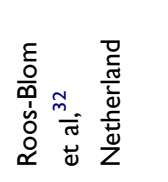 \\
\hline
\end{tabular}


Survey Regarding Pain (NKASRP). ${ }^{20,28,31}$ Knowledge of pain management and barriers to pain control were assessed using the Brockopp-Warden Pain Knowledge/ Bias Questionnaire [BWPKBQ] in two studies. $^{23,25}$ Details regarding the measures used in the selected studies are summarized in Table 2 for quantitative studies and Table 3 for qualitative studies. Meanwhile, Table 1 presents a summary of the 20 reviewed studies.

\section{Strengths and Limitations of the Selected Studies}

Some of the strengths of the selected studies included the use of a large sample size, ${ }^{19,25,28}$ multicenter analysis such as Roos-Blom et al, ${ }^{32}$ and the recruitment of samples from several units. ${ }^{14,27,29,31}$ Also, most of the studies reported using valid instruments to examine the study variables. However, the studies also had various limitations. For example, some studies had limited generalizability due to the use of convenience, consecutive, and purposive sampling techniques. ${ }^{18,19,25,27-29,31,33}$ Also, in ten of the studies, most of the participants were female, and therefore, the results may not be representative of the targeted populations. . $^{14,24,26,29,31,33}$ Other limitations included low response $\operatorname{rates}^{18,19,32}$ and small sample sizes. $^{15,23,26,29,31}$ Additionally, the majority of the selected articles were descriptive correlational studies, which did not allow for causality inference to be established.

\section{Nurse-Related Barriers}

Fifteen of the selected studies reported poor knowledge among nurses regarding critically ill patients' pain as being a barrier to effective pain relief. Eight of the 15 studies reported nurses' lack of knowledge regarding pain assessment, ${ }^{13,14,18,19,25,27,33}$ five reported poor knowledge regarding pain management, ${ }^{20,23,24,29,34}$ and only two reported both. ${ }^{28,31}$ The remaining studies did not discuss the link between nurses' knowledge and pain assessment and management in critically ill adult patients. Deficits in knowledge related to pain assessment and management were most frequently related to (1) behavioral pain indicators (eg facial expressions); ${ }^{17,19,28}$ (2) perception of patient's pain tolerance (eg patient with severe pain cannot be distracted); ${ }^{28,31}$ (3) the use of verbal and nonverbal pain assessment tools; ${ }^{13,14,18,27,33}$ (4) pharmacological pain management, ${ }^{20,23,28,31}$ especially among post-operative patients ${ }^{16}$ and those with terminal illnesses; ${ }^{24}$ and (5) nonpharmacological management. ${ }^{29}$
Among the selected studies, 13 discussed nurses' poor attitudes as being a barrier to effective pain control. Five of these studies reported that the majority of critical care nurses had improper and infrequent use of behavioral pain assessment tools for nonverbal patients. ${ }^{14,18,19,23,27}$ Also, four studies mentioned that nurses underestimate the importance of pain assessment for sedated patients or patients with a decreased level of consciousness. ${ }^{14,18,19,23,27}$ Furthermore, most of the participating critical care nurses relied on measuring physiological parameters or observing odd behaviors when assessing critical care patients' pain rather than using formal pain assessment tools. ${ }^{18,19,26,28,33}$ Moreover, although self-reporting is the gold standard for pain assessment, three studies reported that nurses perceived self-reporting of pain to be an inaccurate measure of pain intensity among critically ill adult patients. ${ }^{24,27,30}$ Another three studies found that nurses perceived there to be no need to assess pain or administer primitive analgesia before carrying out pain-inducing procedures. ${ }^{19,21,30}$

Khalil and Mashaqbeh ${ }^{28}$ reported that the majority of the participating nurses tended to encourage their patients to endure pain as much they could before offering analgesia. The study also reported that patients' spiritual beliefs did not play a significant role in patients' under-reporting of pain. Only Khali ${ }^{29}$ highlighted nurses' misconceptions related to "fear of addiction" and how these misconceptions relate to the issue of pain under treatment or untreatment in critically ill patients. According to Wang and Tsai $^{20}$ and Kizza and Muliira, ${ }^{30}$ the majority of nurses perceived there to be no need for administering analgesics to sedated patients receiving pain-inducing procedures, as nurses perceived these patients to not feel pain. Moreover, in some cases, nurses were found to be unsure about the patient's pain rating, which limited their administration of analgesics. ${ }^{13,27}$ Also, Topolovec-Vranic et al ${ }^{22}$ discussed analgesics misuse as being a barrier to pain management in critically ill patients.

\section{Nurse-Related Facilitators}

A limited number of the reviewed studies discussed improved knowledge among nurses as being a facilitator of optimal pain relief. ${ }^{17,19,27,28,31}$ The most frequently reported facilitators of pain management in adult critically ill patients were (1) better understanding and interpretation of pain behaviors, ${ }^{18,19,27}$ (2) sufficient knowledge of pharmacological interventions, ${ }^{17,28,31}$ and (3) ongoing education and professional training related to pain assessment and management. ${ }^{23,25}$ Furthermore, taking into account 
Table 2 The Summary of the Measures Used in the Selected Quantitative Studies

\begin{tabular}{|c|c|c|c|}
\hline Name of Tool & $\begin{array}{l}\text { Number of Studies } \\
\text { They Appear in }\end{array}$ & Purpose & Psychometric Properties \\
\hline $\begin{array}{l}\text { Nurses' Knowledge and Attitudes } \\
\text { Survey Regarding Pain (NKASRP) }\end{array}$ & 4 & $\begin{array}{l}\text { To evaluate nurses' knowledge of \& } \\
\text { attitudes towards pain assessment \& pain } \\
\text { management. }\end{array}$ & $\begin{array}{l}\text { Cronbach's alpha of } 0.73 \text {, Test-retest } \\
\text { reliability of }>0.80 \text {, Content and } \\
\text { construct validity were established } \\
\text { by pain experts }\end{array}$ \\
\hline $\begin{array}{l}\text { The Pain Assessment and } \\
\text { Management for the Critically III } \\
\text { survey }\end{array}$ & 3 & $\begin{array}{l}\text { To assess nurses' pain assessment practices } \\
\text { among verbal \& nonverbal patients. }\end{array}$ & $\begin{array}{l}\text { Content validity, test-retest } \\
\text { reliability, and comprehensiveness } \\
\text { were tested by ten experts in pain. }\end{array}$ \\
\hline $\begin{array}{l}\text { The Pain Assessment and } \\
\text { Management for the Critically III } \\
\text { survey(modified) }\end{array}$ & I & $\begin{array}{l}\text { To assess nurses' pain assessment practices } \\
\text { among verbal \& nonverbal patients. }\end{array}$ & $\begin{array}{l}\text { Cronbach's alpha of the modified } \\
\text { tool } 0.7 I \text {, content validity of } 0.90\end{array}$ \\
\hline $\begin{array}{l}\text { Brockopp-Warden Pain } \\
\text { Knowledge/Bias Questionnaire } \\
\text { [BWPKBQ]) }\end{array}$ & 2 & $\begin{array}{l}\text { To evaluate nurses' knowledge of pain relief } \\
\& \text { biases towards certain patient groups. }\end{array}$ & $\begin{array}{l}\text { Test and retest correlation } \\
\text { coefficient } 0.86\end{array}$ \\
\hline $\begin{array}{l}\text { Numeric rating scale, Critical } \\
\text { Care Pain Observational Tool }\end{array}$ & I & $\begin{array}{l}\text { To assess pain levels among verbal \& } \\
\text { nonverbal patients, respectively }\end{array}$ & $\begin{array}{l}\text { The authors only mentioned that all } \\
\text { instruments used had been validated }\end{array}$ \\
\hline $\begin{array}{l}\text { The Strengthening the Reporting of } \\
\text { Observational Studies in } \\
\text { Epidemiology EQUATOR checklist }\end{array}$ & 1 & $\begin{array}{l}\text { To evaluate nurses' knowledge of pain } \\
\text { indicators, nurse-physician collaboration \& } \\
\text { satisfaction, \& adequacy of pain management. }\end{array}$ & $\begin{array}{l}\text { Cronbach's alpha of three sections } \\
0.82,0.94,0.84 \text { respectively }\end{array}$ \\
\hline $\begin{array}{l}\text { Nonpharmacological pain } \\
\text { management checklist }\end{array}$ & I & $\begin{array}{l}\text { To explore critical care nurses' } \\
\text { implementation of nonpharmacological pain } \\
\text { interventions to control their patients' pain. }\end{array}$ & $\begin{array}{l}\text { Test and retest correlation } \\
\text { coefficient of } 0.7\end{array}$ \\
\hline $\begin{array}{l}\text { The Staff Satisfaction } \\
\text { Questionnaire and Staff End-of- } \\
\text { Study Questionnaire }\end{array}$ & I & $\begin{array}{l}\text { To investigate pain assessment \& } \\
\text { management from nurses' perspective \& } \\
\text { their confidence level in assessing \& } \\
\text { managing pain in both verbal \& nonverbal } \\
\text { patients. }\end{array}$ & $\begin{array}{l}\text { The authors did not evaluate the } \\
\text { psychometric properties of the tools }\end{array}$ \\
\hline $\begin{array}{l}\text { The Patient Pain Management } \\
\text { Questionnaire }\end{array}$ & I & $\begin{array}{l}\text { To assess patients' pain management from } \\
\text { their point of view \& their level of } \\
\text { satisfaction during their experience in } \\
\text { intensive care units }\end{array}$ & $\begin{array}{l}\text { The authors did not evaluate the } \\
\text { psychometric properties of the tool }\end{array}$ \\
\hline
\end{tabular}

physiological indicators, in addition to verbal and nonverbal indicators, when carrying out pain assessment was found to facilitate pain management in adult critically ill patients. ${ }^{18,19}$ Moreover, improved perception of patients' pain among nurses was found to be another facilitator of pain management. ${ }^{18}$

\section{Patient-Related Barriers and Facilitators}

Despite the relatively large number of research studies examining patient-related barriers to pain assessment and management, only eight of the reviewed studies discussed these barriers from the perspective of critical care nurses. The most frequently reported barrier was patients' inability to communicate, ${ }^{13,18,22}$ followed by hemodynamic instability. ${ }^{18,29}$ Also, it was found that patient history of substance abuse, alcoholism, or suicidal attempts impeded proper pain management. ${ }^{20,23}$ Only five of the 20 studies reported examples of patient-related facilitators of pain assessment and management. ${ }^{13,20,22,24,28}$ The gold standard of pain assessment is self-reporting of pain by the patient. Thus, three studies reported patients' self-reporting of pain as being the most accurate measure of pain and a facilitator of effective pain management. ${ }^{20,24,28}$ In the study of Alasiry et al, ${ }^{13}$ the participants emphasized the importance of using subjective assessment for critically ill conscious patients. With regard to the objective assessment of pain in critical care patients, the most 
Table 3 The Summary of the Measures Used in the Selected Qualitative Studies

\begin{tabular}{|c|c|c|c|}
\hline Authors & Method & Purpose & Type of Analysis \\
\hline Deldar et $\mathrm{al}^{18}$ & Semi-structured interviews & $\begin{array}{l}\text { To investigate a better understanding of the } \\
\text { current challenges faced by critical care } \\
\text { nurses in using pain assessment tools among } \\
\text { nonverbal patients }\end{array}$ & $\begin{array}{l}\text { Inductive approach based on } \\
\text { the Graneheim and Lundman } \\
\text { method. }^{59}\end{array}$ \\
\hline Alasiry et $\mathrm{al}^{13}$ & $\begin{array}{l}\text { Semi-structured, 20-minute } \\
\text { interviews }\end{array}$ & $\begin{array}{l}\text { To gain a better understanding of pain } \\
\text { assessment and pain management in critically } \\
\text { ill patients in cardiac care units from nurses' } \\
\text { perspectives. }\end{array}$ & $\begin{array}{l}\text { Content analysis using manual } \\
\text { analyzing based on the } \\
\text { Graneheim and Lundman } \\
\text { method. }^{59}\end{array}$ \\
\hline Subramanian et al ${ }^{16}$ & $\begin{array}{l}\text { Semi-structured interviews with } \\
\text { nurses using the critical incident } \\
\text { technique. Interviews lasted between } \\
45 \text { minutes- } 1.5 \text { hours }\end{array}$ & $\begin{array}{l}\text { To understand challenges related to effective } \\
\text { pain management among critically ill patients } \\
\text { from nurses' perspective. }\end{array}$ & $\begin{array}{l}\text { Content analysis using the } \\
\text { framework analysis developed } \\
\text { by the National Centre for } \\
\text { Social Research. }\end{array}$ \\
\hline $\begin{array}{l}\text { Lindberg \& } \\
\text { Engström }{ }^{15}\end{array}$ & $\begin{array}{l}\text { Semi-structured interviews lasting } \\
\text { between } 20-70 \text { minutes }\end{array}$ & $\begin{array}{l}\text { To gain a better understanding of critical care } \\
\text { nurses' experiences in assessing and } \\
\text { managing postoperative patients' pain in an } \\
\text { intensive care unit }\end{array}$ & $\begin{array}{l}\text { Content analysis described by } \\
\text { Downe-Wamboldt. }^{61}\end{array}$ \\
\hline
\end{tabular}

commonly reported behavioral pain indicators were motor activity (eg, involuntary movements) and facial activity (eg, facial grimacing). ${ }^{13}$ Even in cases where the patients were unable to verbalize their needs, the nurses used alternative methods in addition to behavioral indicators, such as helping patients write or draw their needs on paper. Moreover, in the study of Pollmann-Mudryj, ${ }^{22}$ the participating nurses reported that if they continued to rely on behavioral indicators only to interpret patients' pain, communication between nurses and patients would be lost.

\section{Physician-Related Barriers and Facilitators}

Only eight of the reviewed studies discussed physicianrelated barriers and enablers of pain management. The most frequently reported barrier was “physicians' prescription of analgesics does not depend on the evaluation of pain scores". ${ }^{18,19,22}$ Also, Deldar et al, ${ }^{14}$ reported that poor interaction between physicians and nurses regarding nonverbal patients' pain impedes effective treatment. Moreover, Subramanian et $\mathrm{al}^{16}$ reported junior doctors' lack of experience and senior doctors' heavy workloads as being significant barriers to effective pain assessment and management. As for the facilitators of pain assessment and management in critically ill adult patients, collaboration between physicians and nurses was the most frequently reported facilitator, ${ }^{13,17}$ followed by "adequate analgesics doses prescribed by physicians". 18,30

\section{System/Organization-Related Barriers}

Of the 20 selected studies, 12 articles discussed barriers to pain management associated with organizational factors. The barriers reported most frequently were (1) lack of standardized pain assessment forms and tools for verbal critically ill patients ${ }^{16,30}$ and nonverbal patients, ${ }^{14,19,20}$ (2) lack of standardized guidelines and protocols for pain evaluation and control, ${ }^{14,16,19,30,32}$ (3) heavy workloads of nurses, ${ }^{14,18,30}$ (4) nursing staff shortages, ${ }^{13,29}$ (5) inadequate nurse-to-patient ratios, ${ }^{14,32}$ (6) inadequate training and education related to pain assessment and management, ${ }^{14,19,22,30}$ (7) nontherapeutic, ineffective, or complicated discussions about pain treatment during medical rounds, ${ }^{18}$ (8) lack of documentation of pain assessment findings, ${ }^{30}$ (9) lack of autonomous decision-making related to pain management, ${ }^{16,20}$ and (10) poor hospital environments, such as shared rooms with only curtains between patients. ${ }^{15}$ Also, only $\operatorname{Hamdan}^{27}$ found a significant relationship between hospital type and adequate pain assessment by nurses, whereby nurses in private and governmental hospitals used pain assessment tools less often than did nurses in educational hospitals.

\section{System/Organization-Related Facilitators}

Only six studies discussed system-related facilitators of pain assessment and management among adult critically ill patients, and the most frequently reported facilitators were (1) continuous education and professional training for critical care 
nurses, ${ }^{13,18-20,28,29}$ and (2) discussing and reporting patients' pain scores during nurse-to-nurse handovers. ${ }^{18,19,30}$ Furthermore, higher hospital accreditation was significantly associated with improved pain assessment practices. ${ }^{20}$ Moreover, Wøien ${ }^{21}$ reported that establishing evidence-based protocols for pain evaluation and documentation led to improved pain control plans. The perceived barriers and facilitators to pain assessment and management in critical care patients are summarized in Table 4.

\section{Discussion}

This review included 20 studies published over the previous 10 years and focused on the barriers and facilitators related to pain assessment and management in critical care units from the perspective of nurses. A limited number of relevant studies were identified. Nurse-related barriers were studied more than other forms of barriers (ie, patientand system-related barriers), with insufficient education and training related to pain assessment among nurses being the most commonly reported nurse-related barrier.
This particular finding is consistent with the findings of a recent systematic review which found nurses' knowledge deficiencies and poor skills to be significant barriers to pain assessment among nursing home residents. ${ }^{35}$

\section{Nurse-Related Barriers and Facilitators}

Given that they spend most of their time caring for patients, nurses play a major role in pain assessment in critically ill patients. ${ }^{36}$ However, limited studies have explored nurse-related barriers to pain assessment. According to the literature review of McAuliffe et al, ${ }^{36}$ nurses have deficient knowledge related to pain assessment in people with dementia (PWD). This is consistent with our review results regarding nurses' lack of knowledge related to pain assessment, as PWD share some characteristics with critically ill patients, such as difficulty self-reporting pain. Also, in the current review, poor knowledge of nurses regarding pain management among ICU patients was found to be a barrier to effective pain relief. Similarly, several previous studies have reported

Table 4 The Barriers to and Facilitators of Pain Assessment and Management

\begin{tabular}{|c|c|c|c|}
\hline \multicolumn{4}{|c|}{ Barriers } \\
\hline Nurse-Related Barriers & $\begin{array}{l}\text { Patient-Related } \\
\text { Barriers }\end{array}$ & Physician-Related Barriers & System-Related Barriers \\
\hline $\begin{array}{l}\text { - Nurses' lack of knowledge regarding } \\
\text { the use of pain assessment tools } \\
\text { - Nurses' poor knowledge regarding } \\
\text { pharmacological pain management } \\
\text { - Nurses' improper and infrequent } \\
\text { use of behavioral pain assessment } \\
\text { tools for nonverbal patients }\end{array}$ & $\begin{array}{l}\text { - Patients' inability to } \\
\text { communicate } \\
\text { - Patients' hemodynamic } \\
\text { instability } \\
\text { - Patients' history of } \\
\text { substance abuse, } \\
\text { alcoholism, or suicidal } \\
\text { attempts }\end{array}$ & $\begin{array}{l}\text { - Physicians' prescription of } \\
\text { analgesics being independent of } \\
\text { evaluation pain scores } \\
\text { - Poor interaction of physicians } \\
\text { with nurses regarding nonverbal } \\
\text { patients' pain } \\
\text { - Junior doctors' lack of } \\
\text { experience and senior doctors' } \\
\text { heavy workloads }\end{array}$ & $\begin{array}{l}\text { - Absence of standardized guidelines } \\
\text { and protocols for pain evaluation and } \\
\text { control } \\
\text { - Inadequate training and education } \\
\text { related to pain assessment and } \\
\text { management } \\
\text { - Lack of standardized pain assessment } \\
\text { forms and tools for verbal and } \\
\text { nonverbal critically ill patients }\end{array}$ \\
\hline \multicolumn{4}{|c|}{ Facilitators } \\
\hline Nurse-Related Facilitators & $\begin{array}{l}\text { Patient-Related } \\
\text { Facilitators }\end{array}$ & $\begin{array}{l}\text { Physician-Related } \\
\text { Facilitators }\end{array}$ & System-related facilitators \\
\hline $\begin{array}{l}\text { - Ongoing education and professional } \\
\text { training related to pain assessment } \\
\text { and management } \\
\text { - Nurses' knowledge regarding pain } \\
\text { behaviors and indicators } \\
\text { - High level of knowledge among } \\
\text { nurses about the pharmacological } \\
\text { management of pain } \\
\text { - Nurses' realization of the } \\
\text { importance of physiological indicators } \\
\text { in pain assessment }\end{array}$ & - Self-reporting of pain & $\begin{array}{l}\text { - Collaboration between } \\
\text { physicians and nurses } \\
\text { Prescription of adequate } \\
\text { analgesics doses by physicians }\end{array}$ & $\begin{array}{l}\text { - Reporting and discussing patients' } \\
\text { pain scores during nurse-to-nurse } \\
\text { handovers } \\
\text { - Continuous education and } \\
\text { professional training for critical care } \\
\text { nurses }\end{array}$ \\
\hline
\end{tabular}


that nurses' lack of knowledge regarding postoperative pain management impeded the achievement of optimal pain control. ${ }^{37}$

A previous systematic review emphasized the importance of using behavioral pain indicators for pain assessment in patients with communication deficits, as opposed to relying only on self-reporting to assess pain. Similar to our findings, the most commonly reported behavioral pain indicators were motor activity (eg involuntary movements) and facial activity (eg facial grimacing). ${ }^{38}$ In our review, nurses were found to misunderstand these behavioral indicators, thus complicating the effective assessment and prompt management of pain in critical care patients. $^{17,19,28}$ This misunderstanding may be due to inconsistency in these behaviors between critical care patients and nurses' lack of knowledge, in addition to poor formal nursing education and clinical training. ${ }^{38}$

Tolerance of pain differs from one person to another. Several factors influence patients' pain tolerance, including emotions and lifestyle factors. ${ }^{39}$ According to Saifan et $\mathrm{al}^{39}$ nurses had several beliefs and misconceptions related to patients' pain tolerance which act as barriers to effective pain management. For example, they believe that patients with severe pain cannot be distracted. These findings support the finding of our review that using both verbal and nonverbal pain assessment tools for critical care patients is essential for optimal pain evaluation because it provides a holistic and comprehensive assessment of pain and allows nurses to feel more confident. By using both verbal and nonverbal pain assessment tools for critical care patients, nurses capture affective and physiological components of the pain experience and become certain regarding suspected pain in critical care patients. ${ }^{19,28}$ Several studies are consistent with our review findings, indicating that nurses' lack of knowledge regarding how to use verbal and nonverbal pain assessment tools is a major barrier to pain relief. ${ }^{40}$ For example, a review of the literature related to pain in PWD found nurses to have poor knowledge and practices related to the use of pain assessment tools. $^{40}$

Adequate administration of pharmacological interventions, specifically opioids, plays an important role in effective pain management. ${ }^{39}$ However, only three of the selected studies reported sufficient knowledge among nurses regarding pharmacological management as being a facilitator of adequate pain relief. Consistent with this finding, several published studies reported nurses' lack of knowledge regarding pharmacological management of pain as being a significant barrier to pain management. ${ }^{40}$ This includes lack of knowledge regarding opioids usage, dosage, and routes of administration. ${ }^{39}$ Moreover, there is limited evidence regarding nurses' knowledge of pharmacological pain management and how it relates to effectively assess and promptly treat pain among critical care patients.

Prolonged use of analgesics has many adverse effects on patients' health status. ${ }^{9}$ For that reason, nonpharmacological interventions may be an effective alternative for pain control among critically ill patients. ${ }^{11}$ There is limited evidence related to nurses' knowledge of and attitudes towards the use of nonpharmacological interventions among adult critically ill patients. In the present review, only one study was found to explore this issue, and this study reported insufficient knowledge among nurses regarding the use of nonpharmacological interventions. ${ }^{29}$ Despite the limited evidence in the literature, there are conflicting findings regarding nurses' levels of knowledge and attitudes related to nonpharmacological interventions in general. According to Puntillo et al, ${ }^{1}$ nurses working in medical and surgical wards had a satisfactory level of knowledge regarding nonpharmacological pain interventions. Conversely, the study of Munkombwe et $\mathrm{al}^{41}$ which was conducted to investigate nurses' palliative care practices, showed nurses to have inadequate knowledge and poor attitudes related to nonpharmacological management.

Nurses' attitudes and beliefs related to pain may influence nurses' provision of proper pain management. ${ }^{2}$ More than half of the reviewed studies reported such attitudes and beliefs as being either facilitators of or barriers to pain assessment and management. For example, although behavioral pain assessment tools have limitations, frequent observing or monitoring of pain behaviors using validated and reliable assessment tools is an essential pain assessment practice in the case of patients who have difficulty self-reporting pain. ${ }^{2}$ However, some of the reviewed studies reported infrequent use of behavioral pain assessment tools by critical care nurses when caring for nonverbal patients. Similarly, Samarkandi ${ }^{42}$ found ICU nurses to have poor attitudes towards behavioral pain assessment. However, these poor attitudes were found to improve after nurses were taught how to use the Critical-Care Pain Observational Tool (CPOT) for nonverbal patients. Also, a scoping review conducted to explore the issue of pain management in pediatric intensive care units reported poor attitudes among nurses regarding the use of valid 
nonverbal pain assessment tools. ${ }^{43}$ Furthermore, several studies conducted in different countries and settings reported negative attitudes among nurses towards pain assessment and management. ${ }^{42}$ Moreover, Devlin et $\mathrm{al}^{11}$ explored the beliefs of nurses regarding sedation and found the misconception that "sedated patients don't feel pain at all" to be a major barrier to adequate pain management. This finding supports the findings of about one-third of the reviewed studies.

In the current review, two studies reported that nurses emphasized monitoring physiological and behavioral indicators as being an important aspect of pain assessment, which facilitates pain management in adult critically ill patients. However, overreliance on measuring physiological parameters or observing odd behaviors rather than using formal pain assessment tools to assess critical care patients' pain was identified as being a major barrier to pain management in the present review. Although physiological parameters such as blood pressure and heart rate can be used to evaluate patients' pain and provide important clues for effective pain assessment, ${ }^{8}$ it is not recommended that formal pain assessment tools are replaced with these parameters when evaluating pain in critically ill adult patients. ${ }^{2}$

A systematic review study conducted to review pain education interventions for nurses working in acute hospital settings reported that different types of education interventions had improved nurses' attitudes towards and knowledge of pain and increased their confidence in their abilities to assess and manage patients' pain. ${ }^{44}$ This finding is consistent with the findings of our review regarding the impact of education programs on improving nurses' knowledge of and attitudes towards pain in adult critically ill patients. However, future interventional research studies are recommended to bridge the knowledge gap in the literature regarding the role of ongoing education and professional training related to pain for critical care nurses as facilitators of pain management.

\section{Patient-Related Barriers and Facilitators}

Pain self-reporting is considered the best way to assess patients' pain, due to the high subjectivity of the pain experience. ${ }^{20}$ Only three of the reviewed studies reported the patient's ability to self-report pain as being a facilitator of pain evaluation. According to Kizza et al, ${ }^{33}$ a significant proportion of nurses do not trust or believe in patients' self-reporting of pain. Two of the studies selected in our review reported that nurses did their best to encourage critically ill conscious patients to self-report their pain using different methods. However, this is not always possible, especially for patients with severe cognitive impairments or communication deficits. ${ }^{20}$ A systematic review conducted to explore cancer patient-related barriers to pain management reported patients' inability to communicate as being one of the major barriers to effective pain management. ${ }^{45}$ Also, several studies reported similar findings among different patient groups and in different clinical settings. ${ }^{46}$ These findings are similar to our finding that the most commonly reported patient-related barrier, as perceived by critical care nurses, was patients' inability to communicate.

Physiological and behavioral indicators are significant pain clues and should be used in addition to other assessment tools to optimize pain management. ${ }^{8}$ However, there is a lack of consistency and specificity regarding the use of these indicators, which may complicate the assessment and management of pain in critically ill patients. Consistent with our finding, Reardon et $\mathrm{al}^{47}$ found hemodynamic instability to be a perceived barrier to pain assessment and management.

Though uncommon, drug addiction during the management of pain may occur. ${ }^{9}$ Therefore, aberrant drug behaviors should be closely monitored to detect addiction and potential drug abuse, especially in patients with a history of substance abuse or alcoholism, when administering opioids to manage pain in such patients. ${ }^{48}$ According to Passik et al, ${ }^{48}$ patients with a history of substance abuse and those receiving opioids have more serious aberrant drug behaviors as compared to patients with no history of drug abuse. Consistent with our review findings, Paice ${ }^{49}$ revealed having a history of misuse of opioids or alcoholism to be a major barrier to pain management in cancer patients.

\section{Physician-Related Barriers and Facilitators}

A recent systematic review, which explored physicianrelated barriers to effective cancer pain management revealed several barriers as perceived by physicians, including deficiencies in knowledge regarding the use of opioids in cancer pain management, fear of addiction, concerns related to the side effects of opioids, inadequate pain assessment, and inadequate analgesics prescription. ${ }^{50}$ In our review, physicians' adequate prescription of analgesics was perceived by nurses to be a facilitator of effective pain management. Also, both our review and the review of Jacobsen et $\mathrm{al}^{50}$ found physicians' workloads and 
improper interpretation of pain scores as being barriers to adequate pain management. Our review found that insufficient interaction between physicians and nurses impedes effective pain treatment, while improved collaboration between physicians and nurses was found to be a major facilitator. Several previous studies have also reported that poor collaboration between nurses and physicians impedes optimal pain relief., $6,51,52$

\section{System-Related Barriers and Facilitators}

The presence of standardized protocols and guidelines for pain evaluation and management is essential for optimal pain management. ${ }^{53}$ Consistently, our review found that the most frequently reported system-related barrier to effective pain management in critical care unit patients was the lack of standardized pain assessment tools and guidelines and pain management protocols. Wøien ${ }^{21}$ also reported that establishing evidence-based protocols for pain evaluation and documentation is essential for facilitating pain control. Similar findings were also reported by a recent integrative review conducted among hospitalized PWD, which found the lack of standardized pain assessment tools and guidelines to be a major barrier to pain management. ${ }^{53}$

Heavy nurses' workloads and high nurse-patient ratios are considered major barriers to optimal pain control. ${ }^{5}$ However, there is limited evidence in the literature related to these barriers and their association with unrelieved pain in critically ill patients. According to previous studies, the quality of care provided to patients suffering from pain differed significantly according to the type of hospital (ie, public or private) ${ }^{54}$ However, in our review, only a single study was found to have explored the association between hospital type and the tools used to assess pain in critically ill patients. ${ }^{27}$

In the current review, it was found that most critical care nurses had a lack of education regarding pain management, and about half of the reviewed studies highlighted the importance of continuous and professional pain education for critical care nurses. Consistently, several previous studies have emphasized the importance of updating nurses' information related to pain assessment and management in verbal and nonverbal patients. ${ }^{54}$ Ineffective or complicated discussions about pain treatment during medical rounds were also identified as being a system-related barrier to effective pain management in this review. Similarly, three previous studies have reported that reporting and discussing the pain scores of patients during nurse-to-nurse handovers facilitates effective pain management. ${ }^{54-56}$

Documentation of pain assessment and management is essential for ensuring the provision of good quality pain control, as it enhances communication between healthcare providers and leads to consistency in assessment data. ${ }^{55,56}$ However, poor nursing documentation of pain assessment findings was identified in this review as being a barrier to pain management. Also, Rafati et $\mathrm{al}^{56}$ revealed that nurses' lack of documentation of postoperative pain management findings contributed to poor patient outcomes. Moreover, although limited studies in our review discussed the role of nurses' autonomous decision-making in pain management, reporting it to be insufficient among critical care unit nurses, several published studies have reported that nurses' lack of autonomous decision-making impedes effective pain management. ${ }^{57}$

Providing adequate levels of privacy in hospital environments has also been found to facilitate nurses' pain assessment and management practices. ${ }^{55,58}$ This finding supports the finding of the current review that issues related to lack of patient privacy, such as shared rooms with no curtains between patients, were significant barriers to pain control among critical care patients. Other systemrelated barriers pertaining to the hospital environment, such as crowded wards, early discharge of patients, and nursing staff shortages were identified in a recent review. ${ }^{58}$

\section{Limitations of the Review}

This review has several limitations. These limitations include the heterogeneity of the selected studies and the limited availability of relevant studies which examine the primary variables. Also, this review included studies published in English only, while there may be other studies written in other languages investigating the main study variables. Also, in comparison to other studies, some of the reviewed studies were conducted on small samples, which limits the generalizability of the findings of these studies. Further, the reviewed studies were conducted in different countries and regions around the world. Therefore, there may have been confounding variables which were not taken into consideration, but which may have impacted the results, therefore impacting the generalizability of the findings. Finally, another limitation is that most of the reviewed studies were cross-sectional and prospective cohort studies, which limits the determination of causality. 


\section{Relevance to Clinical Practice}

The findings of our study could help hospital managers in developing continuous education and staff development training programs on assessing and managing pain for critical care patients. Establishing continuous education, workshops, professional developmental lectures focusing on pain evaluation and treatment for both critical care nurses and physicians, as well as training courses on how to use pain assessment tools and other behavioral pain indicators, especially for nonverbal patients are highly recommended. Also, our findings could be used to develop an evidence-based standard pain management protocol tailored to effectively assess and promptly treat the pain of critical care patients and emphasizing the importance of alternative and complementary medicine of pain.

\section{Conclusion}

Adequate pain control barriers and facilitators among intensive care unit nurses remain insufficiently researched. Effective pain management in critical care units relies on nurses' knowledge of and attitudes towards pain assessment and relief. Also, various barriers to and facilitators of effective pain relief, including patient-related, nurse-related, physician-related, and system-related were identified and examined in the reviewed studies. The most frequently reported barriers in this study included nurses' lack of knowledge regarding the use of pain assessment tools, patients' inability to communicate, physicians' prescription of analgesics being independent of pain scores evaluation, and absence of standardized guidelines and protocols for pain evaluation and control. For the facilitators, the most reported ones include ongoing education and professional training related to pain assessment and management, patients' ability to self-report pain, effective collaboration between physicians and nurses, and productive discussion of patients' pain scores during nurse-to-nurse handovers.

\section{Acknowledgments}

We want to thank the librarians of Jordan University of Science and Technology for their help.

\section{Disclosure}

The authors declare no conflicts of interest for this work.

\section{References}

1. Puntillo KA, Max A, Timsit J-F, et al. Determinants of procedural pain intensity in the intensive care unit. The Europain ${ }^{\circledR}$ study. $\mathrm{Am}$ J Respir Crit Care Med. 2014;189(1):39-47.

2. Barr J, Fraser GL, Puntillo K, et al. Clinical practice guidelines for the management of pain, agitation, and delirium in adult patients in the intensive care unit. Crit Care Med. 2013;41(1):263-306.

3. Dueñas M, Ojeda B, Salazar A, Mico JA, Failde I. A review of chronic pain impact on patients, their social environment and the health care system. J Pain Res. 2016;9:457. doi:10.2147/JPR. S105892

4. Gélinas C. Pain assessment in the critically ill adult: recent evidence and new trends. Intensive Crit Care Nurs. 2016;34:1-11. doi:10.10 16/j.iccn.2016.03.001

5. Alotaibi K, Higgins I, Day J, Chan S. Paediatric pain management: knowledge, attitudes, barriers and facilitators among nurses-integrative review. Int Nurs Rev. 2018;65(4):524-533. doi:10.1111/inr.12465

6. Mamhidir A-G, Sjölund B-M, Fläckman B, Wimo A, Sköldunger A, Engström M. Systematic pain assessment in nursing homes: a cluster-randomized trial using mixed-methods approach. $B M C$ Geriatr. 2017;17(1):1-16. doi:10.1186/s12877-017-0454-z

7. Stamp R, Tucker L, Tohid H, Gray R. Reliability and validity of the critical-care pain observation tool: a rapid synthesis of evidence. J Nurs Meas. 2018;26(2):378-397. doi:10.1891/1061-3749.26.2.378

8. Korving H, Sterkenburg P, Barakova E, Feijs L. Physiological measures of acute and chronic pain within different subject groups: a systematic review. Pain Res Manag. 2020;2020:1-10. doi:10. $1155 / 2020 / 9249465$

9. Berde C, Nurko S. Opioid Side Effects—Mechanism-Based Therapy. Mass Medical Soc; 2008.

10. Gaskin DJ, Richard P. The economic costs of pain in the United States. J Pain. 2012;13(8):715-724. doi:10.1016/j.jpain.2012.03.009

11. Devlin JW, Skrobik Y, Gélinas C, et al. Clinical practice guidelines for the prevention and management of pain, agitation/sedation, delirium, immobility, and sleep disruption in adult patients in the ICU. Crit Care Med. 2018;46(9):e825-e873.

12. Melnyk BM, Fineout-Overholt E. Evidence-Based Practice in Nursing \& Healthcare: A Guide to Best Practice. Lippincott Williams \& Wilkins; 2011.

13. Alasiry S, Löfvenmark C. Nurses'perceptions of pain assessment and pain management for patients with myocardial infarction in a coronary care unit. Middle East J Nurs. 2013;7(5):9-22.

14. Deldar K, Froutan R, Ebadi A. Challenges faced by nurses in using pain assessment scale in patients unable to communicate: a qualitative study. BMC Nurs. 2018;17(1):1-8. doi:10.1186/ s12912-018-0281-3

15. Lindberg J-O, Engström Å. Critical care nurses' experiences:“A good relationship with the patient is a prerequisite for successful pain relief management". Pain Manag Nurs. 2011;12(3):163-172. doi:10.1016/ j.pmn.2010.03.009

16. Subramanian P, Allcock N, James V, Lathlean J. Challenges faced by nurses in managing pain in a critical care setting. J Clin Nurs. 2012;21(9-10):1254-1262. doi:10.1111/j.1365-2702.2011.03789.x

17. Asman O, Slutsker E, Melnikov S. Nurses' perceptions of pain management adequacy in mechanically ventilated patients. $J$ Clin Nurs. 2019;28(15-16):2946-2952. doi:10.1111/jocn.14896

18. Rose L, Haslam L, Dale C, et al. Survey of assessment and management of pain for critically ill adults. Intensive Crit Care Nurs. 2011;27(3):121-128. doi:10.1016/j.iccn.2011.02.001

19. Rose L, Smith O, Gélinas C, et al. Critical care nurses' pain assessment and management practices: a survey in Canada. Am J Crit Care. 2012;21(4):251-259. doi:10.4037/ajcc2012611

20. Wang HL, Tsai YF. Nurses' knowledge and barriers regarding pain management in intensive care units. J Clin Nurs. 2010;19(2122):3188-3196. doi:10.1111/j.1365-2702.2010.03226.x 
21. Wøien H. Movements and trends in intensive care pain treatment and sedation: what matters to the patient? J Clin Nurs. 2020;29(78):1129-1140. doi:10.1111/jocn. 15179

22. Topolovec-Vranic J, Canzian S, Innis J, Pollmann-Mudryj MA, McFarlan AW, Baker AJ. Patient satisfaction and documentation of pain assessments and management after implementing the adult nonverbal pain scale. Am J Crit Care. 2010;19(4):345-354. doi:10.4037/ ajcc2010247

23. Lewis CP, Corley DJ, Lake N, Brockopp D, Moe K. Overcoming barriers to effective pain management: the use of professionally directed small group discussions. Pain Manag Nurs. 2015;16 (2):121-127. doi:10.1016/j.pmn.2014.05.002

24. Machira G, Kariuki H, Martindale L. Impact of an educational pain management programme on nurses' pain knowledge and attitudes in Kenya. Int J Palliat Nurs. 2013;19(7):341-345. doi:10.12968/ ijpn.2013.19.7.341

25. Schreiber JA, Cantrell D, Moe KA, et al. Improving knowledge, assessment, and attitudes related to pain management: evaluation of an intervention. Pain Manag Nurs. 2014;15(2):474-481. doi:10.1016/ j.pmn.2012.12.006

26. Gerber A, Thevoz A-L, Ramelet A-S. Expert clinical reasoning and pain assessment in mechanically ventilated patients: a descriptive study. Aust Crit Care. 2015;28(1):2-8. doi:10.1016/j.aucc.2014. 06.002

27. Hamdan KM. Nurses' assessment practices of pain among critically ill patients. Pain Manag Nurs. 2019;20(5):489-496. doi:10.1016/j. pmn.2019.04.003

28. Khalil H, Mashaqbeh M. Areas of knowledge deficit and misconceptions regarding pain among jordanian nurses. Pain Manag Nurs. 2019;20(6):649-655. doi:10.1016/j.pmn.2019.02.010

29. Khalil NS. Critical care nurses' use of non-pharmacological pain management methods in Egypt. Appl Nurs Res. 2018;44:33-38. doi:10.1016/j.apnr.2018.09.001

30. Kizza I, Muliira J. Nurses' pain assessment practices with critically ill adult patients. Int Nurs Rev. 2015;62(4):573-582. doi:10.1111/ inr. 12218

31. Ufashingabire CM, Nsereko E, Njunwa KJ, Brysiewicz P. Knowledge and attitudes of nurses regarding pain in the intensive care unit patients in Rwanda. Rwanda J. 2016;3(1):21-26. doi:10.4314/rj. v3i1.4F

32. Roos-Blom M-J, Dongelmans D, Stilma W, Spijkstra JJ, de Jonge E, de Keizer N. Association between organizational characteristics and adequate pain management at the intensive care unit. J Crit Care. 2020;56:1-5. doi:10.1016/j.jcrc.2019.11.010

33. Kizza IB, Muliira JK, Kohi TW, Nabirye RC. Nurses' knowledge of the principles of acute pain assessment in critically ill adult patients who are able to self-report. Int J Afr Nurs Sci. 2016;4:20-27. doi:10.1016/j.ijans.2016.02.001

34. Wang J, Xiao LD, He GP, De Bellis A. Family caregiver challenges in dementia care in a country with undeveloped dementia services. J Adv Nurs. 2014;70(6):1369-1380. doi:10.1111/jan.12299

35. Knopp-Sihota JA, Dirk KL, Rachor GS. Factors associated with pain assessment for nursing home residents: a systematic review and meta-synthesis. J Am Med Dir Assoc. 2019;20(7):884-892. e883. doi:10.1016/j.jamda.2019.01.156

36. McAuliffe L, Nay R, O’Donnell M, Fetherstonhaugh D. Pain assessment in older people with dementia: literature review. J Adv Nurs. 2009;65(1):2-10. doi:10.1111/j.1365-2648.2008.04861.x

37. Dessie M, Asichale A, Belayneh T, Enyew H, Hailekiros A. Knowledge and attitudes of Ethiopian nursing staff regarding post-operative pain management: a cross-sectional multicenter study. Patient Relat Outcome Meas. 2019;10:395. doi:10.2147/ PROM.S234521

38. de Knegt NC, Pieper MJ, Lobbezoo F, et al. Behavioral pain indicators in people with intellectual disabilities: a systematic review. J Pain. 2013;14(9):885-896. doi:10.1016/j.jpain.2013.04.016
39. Saifan AR, Bashayreh IH, Al-Ghabeesh SH, et al. Exploring factors among healthcare professionals that inhibit effective pain management in cancer patients. Cent Eur J Nurs Midwifery. 2019;10(1):967. doi:10.15452/CEJNM.2019.10.0003

40. Burns M, McIlfatrick S. Palliative care in dementia: literature review of nurses' knowledge and attitudes towards pain assessment. Int $J$ Palliat Nurs. 2015;21(8):400-407. doi:10.12968/ijpn.20 15.21.8.400

41. Munkombwe WM, Petersson K, Elgán C. Nurses' experiences of providing nonpharmacological pain management in palliative care: a qualitative study. J Clin Nurs. 2020;29(9-10):1643-1652. doi:10.1111/jocn. 15232

42. Samarkandi OA. Knowledge and attitudes of nurses toward pain management. Saudi J Anaesth. 2018;12(2):220. doi:10.4103/sja. SJA_587_17

43. Ismail A, Forgeron $\mathrm{P}$, Polomeno V, Gharaibeh $\mathrm{H}$, Dagg W, Harrison D. Pain management interventions in the paediatric intensive care unit: a scoping review. Intensive Crit Care Nurs. 2019;54:96-105. doi:10.1016/j.iccn.2019.05.002

44. Drake G, Williams A. Nursing education interventions for managing acute pain in hospital settings: a systematic review of clinical outcomes and teaching methods. Pain Manag Nurs. 2017;18(1):3-15. doi:10.1016/j.pmn.2016.11.001

45. Jacobsen R, Møldrup C, Christrup L, Sjøgren P. Patient-related barriers to cancer pain management: a systematic exploratory review. Scand J Caring Sci. 2009;23(1):190-208. doi:10.1111/j.14716712.2008.00601.x

46. Dequeker S, Van Lancker A, Van Hecke A. Hospitalized patients' vs. nurses' assessments of pain intensity and barriers to pain management. J Adv Nurs. 2018;74(1):160-171. doi:10.1111/jan.13395

47. Reardon DP, Anger KE, Szumita PM. Pathophysiology, assessment, and management of pain in critically ill adults. Am J Health Syst Pharm. 2015;72(18):1531-1543. doi:10.2146/ajhp140541

48. Passik SD, Kirsh KL, Donaghy KB, Portenoy RK. Pain and aberrant drug-related behaviors in medically ill patients with and without histories of substance abuse. Clin J Pain. 2006;22(2):173-181. doi:10.1097/01.ajp.0000161525.48245.aa

49. Paice JA. Cancer pain management and the opioid crisis in America: how to preserve hard-earned gains in improving the quality of cancer pain management. Cancer. 2018;124(12):2491-2497. doi:10.1002/ cncr.31303

50. Jacobsen R, Sjogren P, Moldrup C, Christrup L. Physician-related barriers to cancer pain management with opioid analgesics: a systematic review. J Opioid Manag. 2007;3(4):207-214. doi:10. 5055/jom.2007.0006

51. Ozawa M, Yokoo K. Pain management of neonatal intensive care units in Japan. Acta Paediatr. 2013;102(4):366-372. doi:10.1111/ apa. 12160

52. Ishikawa H, Kawagoe K, Kashiwagi M, Yano E. Nurse-physician collaboration in pain management for terminally ill cancer patients treated at home in Japan. J Palliat Care. 2007;23(4):255-261. doi: $10.1177 / 082585970702300403$

53. Tsai I-P, Jeong -SY-S, Hunter S. Pain assessment and management for older patients with dementia in hospitals: an integrative literature review. Pain Manag Nurs. 2018;19(1):54-71. doi:10.1016/j.pmn. 2017.10.001

54. Tse MMY, Ho SS. Enhancing knowledge and attitudes in pain management: a pain management education program for nursing home staff. Pain Manag Nurs. 2014;15(1):2-11. doi:10.1016/j.pmn.2012.03.009

55. Mędrzycka-Dąbrowska WA, Dąbrowski S, Basiński A. Perception of barriers to postoperative pain management in elderly patients in Polish hospitals-a multicentre study. J Nurs Manag. 2016;24 (8):1049-1059. doi:10.1111/jonm.12405

56. Rafati F, Soltaninejad M, Aflatoonian MR, Mashayekhi F. Postoperative pain: management and documentation by Iranian nurses. Mater Sociomed. 2016;28(1):36. doi:10.5455/msm.2016.28.36-40 
57. Rababa M, Al-Rawashdeh S. Critical care nurses' critical thinking and decision making related to pain management. Intensive Crit Care Nurs. 2021;63:103000. doi:10.1016/j.iccn.2020.103000

58. Taylor A, Stanbury L. A review of postoperative pain management and the challenges. Curr Anaesth Crit Care. 2009;20(4):188-194. doi:10.1016/j.cacc.2009.02.003

59. Graneheim UH, Lundman B. Qualitative content analysis in nursing research: concepts, procedures and measures to achieve trustworthiness. Nurse Educ Today. 2004;24(2):105-112. doi:10.1016/ j.nedt.2003.10.001
60. Ritchie J, Spencer L. Qualitative data analysis for applied policy research. In: Bryman A, Burgess R, editors. Analysing Qualitative Data. London: Routledge; 1994:172-194.

61. Downe-Wamboldt B. Content analysis: method, applications, and issues. Health Care Women Int. 1992;13(3):313-321. doi:10.1080/ 07399339209516006

62. Liberati A, Altman D, Tetzlaff J, et al. The PRISMA statement for reporting systematic reviews and meta-analyses of studies that evaluate health care interventions: explanation and elaboration. Journal of Clinical Epidemiology. 2009;62(10):e1-e34.

\section{Publish your work in this journal}

The Journal of Pain Research is an international, peer reviewed, open access, online journal that welcomes laboratory and clinical findings in the fields of pain research and the prevention and management of pain. Original research, reviews, symposium reports, hypothesis formation and commentaries are all considered for publication. The manuscript

Submit your manuscript here: https://www.dovepress.com/journal-of-pain-research-journal management system is completely online and includes a very quick and fair peer-review system, which is all easy to use. Visit http:// www.dovepress.com/testimonials.php to read real quotes from published authors. 